\title{
The Effect of Organ Motion and Image Artifacts on Monomodal Volume Registration
}

\author{
M van Herk, JC de Munck, MTJG Groenewegen, AR Peters and A Touw \\ Radiotherapy Department, The Netherlands Cancer Institute/Antoni van Leeuwenhoek Huis \\ Plesmanlaan 121, 1066 CX Amsterdam, the Netherlands \\ portal@nki.nl
}

\begin{abstract}
Volume registration, by means of minimizing rms pixel value differences, was tested on pairs of pelvic CT scans in a perturbation experiment to determine its robustness. The tests included registration of 9 scans to themselves and registration of 17 clinical image pairs. Local image artifacts have no influence on performance, but global distortions and organ movement severely degraded the reproducibility. By limiting the algorithm to the gray value range of bone the accuracy is improved at the cost of reliability.
\end{abstract}

\section{Introduction}

Image registration plays an increasingly important role in many medical procedures. The aim of this study is to evaluate the performance of a "standard" automatic volume registration technique for registering pairs of CT scans and to test the sensitivity of the registration algorithm for image artifacts that might occur in clinical practice.

\section{Material and Methods}

We used 17 CT scan pairs from eight prostate cancer patients. The rms difference of pixel values between the two scans was minimized by rigid 3-D translation and rotation. Pixels outside the region of overlap of both scans were ignored. Matching was started from random transformations, and the average displacement of pixels in a region of interest compared to a ground truth match was used to measure the performance. Displacements larger than $5 \mathrm{~mm}$ are counted as failures. The accuracy is defined as the average displacement of all non-failed matches. In the first, identical data experiments, several image artifacts (e.g., holes, noise, cropping, and distortions) were introduced into copies of the same scan that were then matched. In the second, different data experiments, the planning CT of all eight patients was edited manually using a simple paintbrush program to delete selected structures on all slices. The edited scans were registered with unmodified follow up scans. The ground truth was derived using the same registration technique, i.e., only the reproducibility is measured. 


\section{Results}

In the identical data experiments, the match reliability was typically $95 \%$ or more, and the introduced artifacts had little influence. The reliability seemed to increase slightly by blurring or adding noise. Cropping of the data if feet-head direction to $1 / 3$ reduced the reliability to $85 \%$. A reduced accuracy was observed by introducing geometrical distortions or simulating patient movement.

In the different data experiments, the match results were consistently different from the ground truth match, i.e., there existed a significant systematic error component. An accuracy of better than $1 \mathrm{~mm}$ and a visually correct result is obtained only when registration is limited to pixels that contain bone tissue. However, this procedure results in an extremely poor reliability of $13-15 \%$. The small width of the bony structures and associated small overlap for starting positions more than a few $\mathrm{mm}$ away from the correct match may be the cause of this problem. The next best performance $(2 \mathrm{~mm}$ reproducibility and $94 \%$ reliability) is obtained when excluding air pixels and removing the table. The influence of the air pixels is probably due to the significant distortions of the skin surface due to normal day to day differences in bladder filling. Removing bowel contrast or the (mobile) femurs had no effect (Fig. 1). Better results were obtained using surface registration of the segmented pelvis.

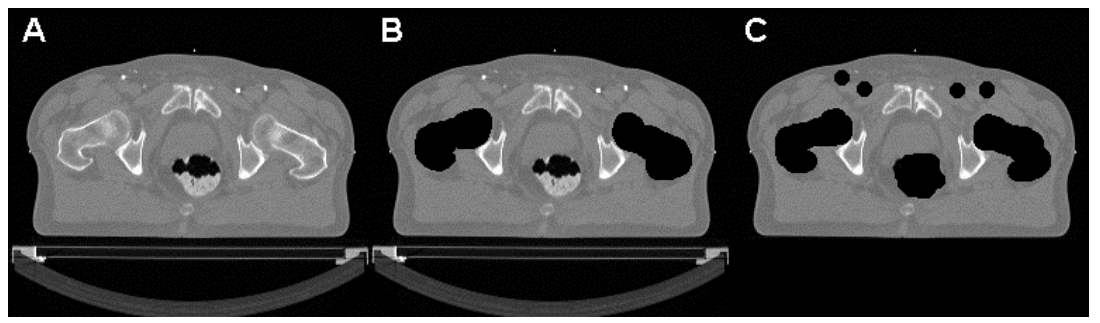

Figure 1. Example of the manual editing process applied in the tests with different data to 17 scan pairs. A) Original slice. B) Femurs removed. The painted region is excluded from the cost function evaluation. C) Bowel (middle) and lymph node (top) contrast removed. Also the table (bottom) was removed. These adaptations hardly improve rms volume registration.

\section{Conclusions}

Volume registration by minimizing rms pixel value differences is highly robust for local image artifacts. However, the accuracy decreases in the presence of global distortions and organ motion. For this reason, volume registration is not very well suited for accurate rigid registration of pelvic CT. The best results in terms of accuracy, but with a poor reliability, are obtained when limiting the registration to bone pixels only. The next best results (about $2 \mathrm{~mm}$ accuracy and 90\% reliability) are obtained when excluding air pixels and the table structure. Bowel contrast and the presence of femur movement are not responsible for the poor performance. 